\title{
Role of Calcitonin Gene-Related Peptide in Light-Aversive Behavior: Implications for Migraine
}

\author{
Ana Recober, ${ }^{1}$ Adisa Kuburas, ${ }^{2}$ Zhongming Zhang, ${ }^{2}$ John A. Wemmie, ${ }^{3,5}$ Michael G. Anderson, ${ }^{2,4}$ and Andrew F. Russo ${ }^{2}$ \\ Departments of ${ }^{1}$ Neurology, ${ }^{2}$ Molecular Physiology and Biophysics, ${ }^{3}$ Psychiatry, and ${ }^{4}$ Ophthalmology and Visual Sciences, University of Iowa, and \\ ${ }^{5}$ Department of Veteran's Affairs Medical Center, Iowa City, Iowa 52242
}

\begin{abstract}
Migraine is a chronic neurological disorder characterized by recurrent episodes of severe unilateral throbbing head pain and associated symptoms, such as photophobia. Our current understanding of the mechanisms underlying migraine has been hampered by limitations in ascertaining migraine symptoms in animal models. Clinical studies have established the neuropeptide calcitonin gene-related peptide (CGRP) as a key player in migraine. Here, we establish a genetic model of photophobia by engineering increased sensitivity to CGRP in mice. These transgenic mice (nestin/hRAMP1) display light-aversive behavior that is greatly enhanced by intracerebroventricular injection of CGRP and blocked by coadministration of the CGRP receptor antagonist olcegepant. This behavior appears to be an indicator of photophobia and cannot be fully explained by gross abnormality of ocular anatomy or differences in general anxiety or motor activity. Our findings demonstrate that a single gene, receptor activity-modifying protein 1 (RAMP1), can be a modifier of photophobia and, by extension, suggest that genetic or epigenetic modulation of RAMP1 levels may contribute to migraine susceptibility. Moreover, they validate CGRP hypersensitive mice as a tool for exploring the neurobiology and novel therapies for migraine and other disorders involving photophobia.
\end{abstract}

\section{Introduction}

Migraine is a complex neurovascular disorder (Goadsby et al., 2002) that has a major impact on the patient and society (Lipton et al., 2007). Although the field of migraine pathophysiology has experienced decisive advances in the past decades, there are still many unanswered questions, in part attributable to the lack of appropriate animal models. A key player in migraine pathophysiology is the neuropeptide calcitonin gene-related peptide (CGRP) (Arulmani et al., 2004). Plasma levels of CGRP have been found to be elevated during migraine attacks (Goadsby et al., 1990; Goadsby and Edvinsson, 1993; Juhasz et al., 2005), although this is controversial (Tvedskov et al., 2005; Benemei et al., 2007; Tfelt-Hansen and Le, 2009). Importantly, individuals with migraine (Lassen et al., 2002) but not healthy controls (Petersen et al., 2005) develop a migraine-like headache in response to intravenous administration of CGRP, suggesting that migraineurs are especially sensitive to CGRP. Finally, CGRP recep-

\footnotetext{
Received April 9, 2009; revised May 14, 2009; accepted June 1, 2009.

This work was supported by the National Headache Foundation and National Institutes of Health Grants DE016511, DE018149, and T32 NS045449. We thank Pedro Gonzalez-Alegre for invaluable suggestions and use of his video tracking system in early studies, Eric Kaiser and Eric van Otterloo for initial development of the lightaversion assay, Jacqueline N. Crawley for her suggestions on the light-aversion assay, Khristofor Agassandian for his help obtaining (SF samples, Bengt von Mentzer (Pharmnovo) for providing olcegepant, Kai Wang for his help with some of the statistical analysis, and Donna Hammond and members of the Russo laboratory for stimulating discussions. A.R. designed, performed, and analyzed the experiments and wrote this manuscript. A.K. assisted with all the experiments. Z.Z. generated the transgenic mice and performed the CSF experiments. J.A.W. and M.G.A. provided advice and assistance with the anxiety and visual assays, respectively, and edited this manuscript. A.F.R. conceived the idea of testing photophobia in RAMP1 mice, directed the experiments, and helped write this manuscript.

Correspondence should be addressed to Dr. Andrew F. Russo, Department of Molecular Physiology and Biophysics, Carver College of Medicine, University of lowa, 5-432 BSB, 51 Newton Road, lowa City, IA 52242. E-mail: andrew-russo@uiowa.edu.

DOI:10.1523/JNEUROSCI.1727-09.2009

Copyright $\odot 2009$ Society for Neuroscience $\quad 0270-6474 / 09 / 298798-07 \$ 15.00 / 0$
}

tor antagonists are effective at alleviating the pain and associated symptoms of a migraine attack (Olesen et al., 2004; Ho et al., 2008).

Photophobia leading to light-aversive behavior is commonly reported by migraineurs during acute attacks and, to a lesser degree, between attacks (Mulleners et al., 2001). Photophobia also accompanies other less frequent but likewise disabling conditions, such as cluster headache and other trigeminal autonomic cephalalgias and blepharospasm (Goadsby and Edvinsson, 1994; Bahra et al., 2002; Hallett et al., 2008; Irimia et al., 2008). The mechanisms underlying photophobia are not known; however, it seems likely that there is involvement of the trigeminal system (Drummond and Woodhouse, 1993; Kowacs et al., 2001; Okamoto et al., 2009). Photophobia in blind patients suggests contributions from a nonvisual pathway (Amini et al., 2006). In addition, trigeminal autonomic cephalalgias, a less common group of primary headache disorders, are characterized by unilateral trigeminal-mediated pain frequently associated with ipsilateral photophobia (Irimia et al., 2008). As a major neuropeptide in the trigeminal system, CGRP is likely to be involved in the development of photophobia, but to date, this has not been directly tested.

To test the role of CGRP in photophobia and determine whether heightened CGRP sensitivity leads to migraineassociated symptoms in mice, we developed an experimental model of photophobia-related behavior using conditional transgenic mice with elevated expression of human receptor activitymodifying protein 1 (hRAMP1). RAMP1 is a required subunit of the CGRP receptor that is necessary for trafficking to the cell surface and ligand-binding specificity (McLatchie et al., 1998). RAMP1 is also responsible for the species selectivity of the CGRP 
receptor antagonists being used in clinical studies (Mallee et al., 2002). Nestin/hRAMP1 mice selectively express human RAMP1 in nervous tissues. These are double transgenic mice that express hRAMP1 after conditional removal of an upstream stop sequence by Cre recombinase under control of the nestin promoter (Zhang et al., 2007). Nestin/hRAMP1 mice have twofold greater levels of total mouse and human RAMP1 in the peripheral nervous system and CNS and an increased neurogenic inflammatory response to CGRP (Zhang et al., 2007). In this study, we have used nestin/ hRAMP1 mice to demonstrate that increased sensitivity to CGRP leads to light-aversive behavior in mice, which we propose to be analogous to photophobia in migraine patients.

\section{Materials and Methods \\ Animals}

As specified throughout the text, two strains of nestin/hRAMP1 mice with differing genetic background were used, both with identical $\mathrm{Tg}$ (Nescre) $1 \mathrm{Kln} / \mathrm{J}$ and $\mathrm{Tg}(\mathrm{RAMP1})$ alleles (Tronche et al., 1999; Zhang et al., 2007). For STOCK $T g$ (Nes-cre) $1 K \ln T g(R A M P 1)$, abbreviated as nestin/ hRAMP1, Nes-cre was introduced by an intercross involving a B6;129 mixed genetic background, yielding mice with contributions from the B6, 129, and SJL genetic backgrounds. For B6;SJL-Tg(Nes-cre $) 1 \mathrm{Kln}$ $\operatorname{Tg}(R A M P 1)$, abbreviated as nestinJ/hRAMP1, Nes-cre was introduced by an intercross involving mice obtained from The Jackson Laboratory (stock 003771) and only the B6 genetic background, yielding mice with contributions from the B6 and SJL genetic backgrounds. Control mice were littermates nontransgenic $(\mathrm{R}-/ \mathrm{C}-)$ or single transgenic (not expressing hRAMP1) nestin-cre $(\mathrm{R}-/ \mathrm{C}+)$ or $\mathrm{Cx} 1$-green fluorescent protein (GFP)-hRAMP1 $(\mathrm{R}+/ \mathrm{C}-)$. All mice were used only once, except those tested in the open field (uninjected), which were subsequently tested in the predator odor-evoked fear assay (uninjected). For the lightaversion assay, all mice were 10-20 weeks old. For the open field and predator odor-evoked fear assay, all mice were $8-10$ weeks old. Animal care and procedures were approved by the University of Iowa Animal Care and Use Committee and performed in accordance with the standards set by the National Institutes of Health.

\section{Drugs}

Rat CGRP (Sigma) was diluted in PBS $(0.5 \mathrm{nmol})$. Olcegepant (BIBN4096BS) was diluted in PBS and 2.5\% DMSO (0.5 nmol). Inhaled 3\% isoflurane was used for brief anesthesia during intracerebroventricular injections. Drug administration was by direct injection in the right lateral ventricle through the intact scalp aiming at $1 \mathrm{~mm}$ posterior to bregma and $1 \mathrm{~mm}$ right from the midline. All the injections were performed by the same person (A.R.) after a period of training yielding a success rate of $89 \%$ ( 24 of 27 mice) demonstrated by injections of dye in the ventricles. The avoidance of deep anesthesia and stereotaxic guidance was important to allow quick recovery of the mice and appropriate performance in the behavioral tests. We used an injector made with a 30 -gauge needle connected by a polyethylene tube to a microsyringe. A $1 \mathrm{~cm}$ polyethylene tube was fitted to the needle to control the depth of the injection $(2.5 \mathrm{~mm})$.

\section{Behavior assays}

Light-aversion test. Mice were individually tested in a custom-made lightaversion chamber [60 length $(\mathrm{L}) \times 60$ width $(\mathrm{W}) \times 45$ height $(\mathrm{H}) \mathrm{cm}$ ] with two equally sized compartments: one brightly lit [1000 lux, thermalneutral fiber optic source (Fiber-lite)], painted white and lacking a top, the other not lit, painted black, and fully enclosed. A small opening $(7 \times$ $7 \mathrm{~cm}$ ) connects the two compartments. This test differs from the originally described light-dark box (Crawley and Goodwin, 1980) by using a more intense light source that does not produce heat. Before testing, mice were acclimated at least $1 \mathrm{~h}$ in their cages in the testing room with the lights on (200 lux inside the cage). The light-aversion chamber was thoroughly cleaned with $95 \%$ EtOH, between animals. Each mouse was gently held on the investigator's palm with minimal tail restrain and placed in the center of the open side of the box, facing away from the dark side. A video tracking system (View Point Life Sciences) was used to monitor behavior and to quantify the total time that mice spent in the
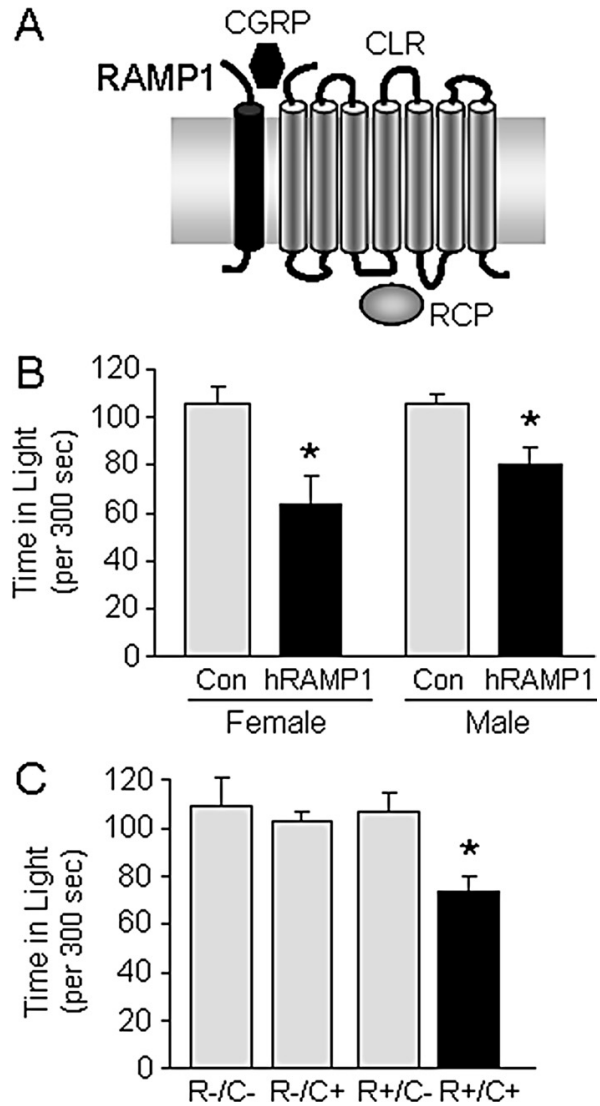

Figure 1. Light-aversive behavior in nestin/hRAMP1 mice. $A$, Schematic of CGRP receptor ( $C L R$, calcitonin-like receptor; $R C P$, receptor component protein). $\boldsymbol{B}$, Total time spent in light by female nestin/hRAMP1 mice $(n=14)$ compared with female control littermates (Con; $n=26$; $\left.{ }^{*} p=0.0147\right)$ and male nestin/hRAMP1 mice $(n=22)$ compared with male control littermates (Con; $\left.n=29 ;{ }^{*} p=0.0358\right)$. C, Total time spent in light by nestin/hRAMP1 mice $(\mathrm{R}+/ \mathrm{C}+; n=$ 36) compared with control littermates (open columns) of all three possible genotypes: nontransgenic $\left(\mathrm{R}-/ \mathrm{C}-; n=14 ;{ }^{*} p=0.0119\right)$, single transgenic nestin-cre $(\mathrm{R}-/ \mathrm{C}+; n=26$; $\left.{ }^{*} p=0.0018\right)$, single transgenic CX1-GFP-hRAMP1 $\left(\mathrm{R}+/ \mathrm{C}-; n=15 ;{ }^{*} p=0.0124\right)$ (hRAMP1, R; cre, C). Error bars indicate SEM.

light versus the dark compartment during $300 \mathrm{~s}$ monitoring intervals. Tests were done in untreated mice and $30 \mathrm{~min}$ after intracerebroventricular injection of $2 \mu \mathrm{l}$ of CGRP ( $0.5 \mathrm{nmol})$, CGRP with olcegepant $(0.5$ nmol each), or vehicle. Mice were briefly anesthetized with $3 \%$ isoflurane during the intracerebroventricular injections and allowed to recover in individual cages for $30 \mathrm{~min}$. Mice were excluded if they showed signs of distress after the injection that interfered with the behavioral assay; this was defined as spending $>90 \%$ of the time inactive in the light compartment of the chamber (automatically determined by the video tracking system). Four out of 100 mice were excluded.

Predator odor-evoked fear test. Mice were placed individually in an acrylic glass chamber $(18 \mathrm{~W} \times 18 \mathrm{~L} \times 18 \mathrm{H} \mathrm{cm})$ with a beaker containing tissue wipes with $30 \mu \mathrm{l}$ of trimethylthiazoline (TMT) (PheroTech) and videotaped for $10 \mathrm{~min}$. Videos were watched, and fear-related freezing behavior was recorded for each minute. Freezing was defined as the absence of movement except for respiration. The test was performed at baseline (no intracerebroventricular injection) and $30 \mathrm{~min}$ after intracerebroventricular injection of CGRP or vehicle as done for the light-aversion test.

Open-field test. Mice were tested for $30 \mathrm{~min}$ in a $40.6 \mathrm{~W} \times 40.6 \mathrm{~L} \times$ $36.8 \mathrm{H} \mathrm{cm}$ open field (San Diego Instruments). A 60 watt light bulb was placed over the open field (400 lux). Time spent in center is measured as a percentage relative to time spent in periphery (periphery was set as 5 $\mathrm{cm}$ ). Activity was defined as total number of beam breaks in the field. The test was performed at baseline (no intracerebroventricular injection) and $30 \mathrm{~min}$ after intracerebroventricular injection of CGRP or vehicle as done for the light-aversion test. 
A
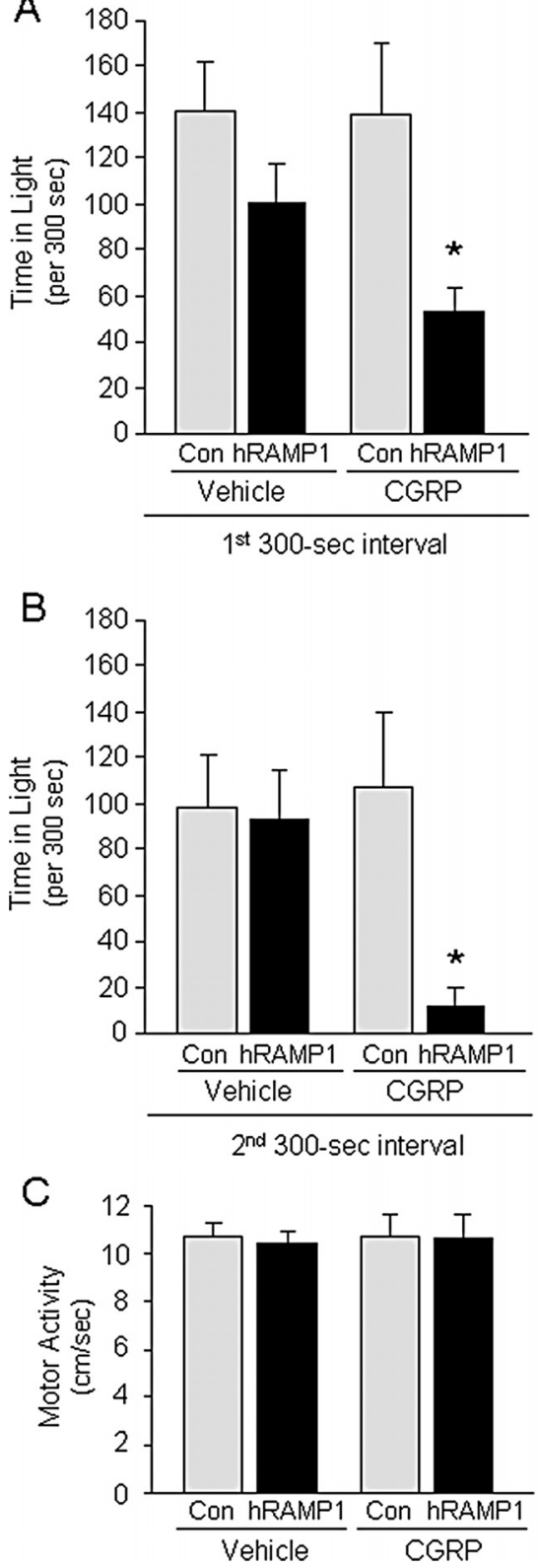
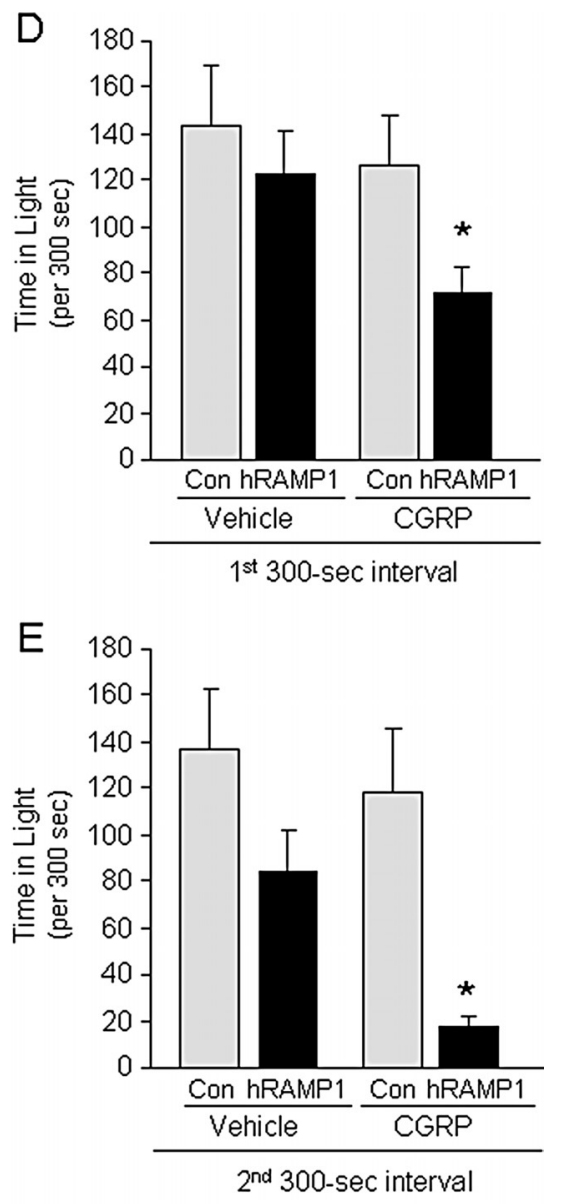

Figure 2. CGRP-induced light-aversive behavior in nestin/hRAMP1 and nestinJ/hRAMP1 mice.A, Total time spent in light in the first 300 s interval by nestin/hRAMP1 mice after intracerebroventricular CGRP ( $\mathrm{hRAMP1;} n=10$ ) compared with vehicle (hRAMP1; $\left.n=8 ;{ }^{*} p=0.0117\right)$ and compared with control (Con) littermates after intracerebroventricular CGRP $\left(n=11 ;{ }^{*} p=0.0221\right) . \boldsymbol{B}$, Total time spent in light in the second 300 s interval by nestin/hRAMP1 mice after intracerebroventricular CGRP (hRAMP1; $n=10)$ compared with vehicle (hRAMP1; $n=8 ;{ }^{*} p=0.0009$ ) and compared with control littermates after intracerebroventricular CGRP (Con; $\left.n=11 ;{ }^{*} p=0.0313\right)$.C, Spontaneous motor activity (centimeters per second) in light-aversion chamber by nestin/hRAMP1 mice after intracerebroventricular CGRP (hRAMP1; $n=10$ ) or vehicle (hRAMP1; $n=8$ ) and control littermates after intracerebroventricular CGRP (Con; $n=11$ ) or vehicle (Con; $n=8)$. $\boldsymbol{D}$, Total time spent in light in the first 300 s interval by nestinJ/hRAMP1 mice after intracerebroventricular vehicle ( $\mathrm{hRAMP1} ; n=4)$ compared with CGRP ( $\mathrm{hRAMP1} ; n=11 ;{ }^{*} p=0.0158$ ) and compared with control littermates after intracerebroventricular CGRP (Con; $\left.n=11 ;{ }^{*} p=0.0087\right)$. $\boldsymbol{E}$, Total time spent in light in the second 300 s interval by nestinJ/hRAMP1 mice after intracerebroventricular vehicle (hRAMP1; $n=4)$ compared with CGRP (hRAMP1; $n=$ $\left.11 ;{ }^{*} p=0.0050\right)$ and compared with control littermates after intracerebroventricular CGRP (Con; $\left.n=11 ;{ }^{*} p=0.0025\right)$. Error bars indicate SEM.

Slit-lamp examination. Eyes were examined with a slit-lamp biomicroscope and photographed with a $40 \times$ objective lens.

\section{CSF measurements}

Mice were anesthetized with intraperitoneal ketamine/xylaxine (87.5/ $12.5 \mathrm{mg} / \mathrm{Kg}$ ). Between 5 and $8 \mu \mathrm{l}$ of CSF were collected by doing a midline scalp incision and introducing a capillary in the cisterna magna. An enzyme immunoassay (SPI-Bio; Cayman Chemical) was used to measure levels of CGRP in CSF.

\section{Statistical analysis}

For the light-aversion studies, we used the twotailed unpaired Mann-Whitney test to assess two-sample comparisons and the KruskalWallis test for multiple comparisons followed by Mann-Whitney test for the relevant comparisons. Two-tailed unpaired Student's $t$ test was used for CSF determinations. ANOVA was used to analyze the anxiety related behavior and motility studies. $p<0.05$ was considered significant. Error bars indicate SEM.

\section{Results}

Nestin/hRAMP1 mice display lightaversive behavior at baseline

To measure light aversion, we used an exploratory assay in which mice have the choice of being in the light or dark area of a chamber. Time spent in the light was compared between nestin/hRAMP1 mice and control littermates at baseline (untreated) conditions. Nestin/hRAMP1 mice have elevated nervous system expression of the hRAMP1 subunit of the CGRP receptor (Fig. 1A). In the absence of treatment, nestin/hRAMP1 mice spent $30 \%$ less time in the light $(73.7 \pm 6.7 \mathrm{~s}$, mean \pm SEM) than control littermates (105.5 $\pm 4.2 \mathrm{~s}$, mean \pm SEM; $p=0.0002)$. This was observed in both male and female mice (Fig. $1 B$ ). The difference between nestin/hRAMP1 mice and control littermates was observed when compared with either the nontransgenic or the two single transgenic control littermates not expressing $h R A M P 1$ (Fig. 1C). After establishing that there were no differences based on sex or control genotypes, both sexes and the three control genotypes were pooled in subsequent analyses.

Ophthalmic slit-lamp examinations demonstrated normal-appearing eyes in nestin/hRAMP1 and control littermates (supplemental Fig. 1, available at www. jneurosci.org as supplemental material).

\section{Nestin/hRAMP1 mice have higher levels of CGRP in CSF}

A potential mechanism underlying this baseline enhanced light aversion could be elevated levels of endogenous CGRP as a result of hRAMP1 expression. To test this hypothesis, we measured CGRP levels in CSF in the mice. We found that CGRP levels were significantly higher in CSF of nestin/ hRAMP1 mice $(n=6 ; 815.8 \pm 189.5 \mathrm{pg} / \mathrm{ml}$, mean \pm SEM) compared with control littermates $(n=6 ; 485.1 \pm 174.3 \mathrm{pg} / \mathrm{ml}$, mean \pm SEM; $p=0.037)$.

\section{Exogenous CGRP enhances light aversion in} nestin/hRAMP1 mice

We then tested the effect of exogenous CGRP, since acute elevations of CGRP during migraine attacks have been reported in 
some, although not all, studies (Benemei et al., 2007; TfeltHansen and Le, 2009). CGRP-treated nestin/hRAMP1 mice showed a significant increase in light aversion (Fig. $2 A, B$ ). During the first $300 \mathrm{~s}$ testing interval, nestin/hRAMP1 mice spent $\sim 50 \%$ less time in the light after intracerebroventricular CGRP compared with vehicle and control mice (Fig. 2A). To identify possible effects of acclimation to the chamber, we doubled the duration of the test and analyzed the behavior separately between the first and second $300 \mathrm{~s}$ intervals. As expected, all groups of mice spent less time exploring the more aversive side in the second interval. Even more striking, though, was the light-aversive effect of CGRP in nestin/hRAMP1 mice in the second 300 s interval. Nestin/hRAMP1 mice spent $>85 \%$ less time in the light during the second interval (Fig. $2 B$ ).

CGRP had other general effects that were observed in all genotypes while recovering from the injection in their cages. These were decreased escape response, decreased exploration, piloerection, and diarrhea. Potential effect of CGRP in spontaneous motility was of particular concern, because it can be a confounding factor in the light-dark box (Crawley and Goodwin, 1980). We analyzed spontaneous motor activity during the light-aversion test and found no differences between the groups (Fig. 2C). A caveat to this result is that the video tracking system only allowed us to evaluate movements in the open side of the chamber and not in the dark.

A different strain of nestin-cre mice (nestinJ/hRAMP1) was used to confirm the contribution of nestin-cre driven hRAMP1 expression to the phenotype independent of the genetic context. The same results obtained with nestin/hRAMP1 mice (Fig. 2A,B) were replicated with nestinJ/hRAMP1 mice (Fig. $2 D, E$ ).

\section{CGRP receptor antagonist prevents CGRP-induced}

light-aversive behavior

To demonstrate that the CGRP-induced light aversion was mediated by the CGRP receptor, we used the CGRP receptor antagonist olcegepant (BIBN-4096BS). This antagonist was selected because of its high affinity for the human CGRP receptor (Doods et al., 2000) and its proven clinical effectiveness to alleviate photophobia and other migraine symptoms in humans during a migraine attack (Olesen et al., 2004). Simultaneous intracerebroventricular injection of the CGRP receptor antagonist olcegepant $(0.5 \mathrm{nmol})$ with CGRP $(0.5 \mathrm{nmol})$ prevented the CGRP-induced light aversion (Fig. 3). Olcegepant had no apparent effect when injected alone (Fig. 3). Furthermore, CGRP and olcegepant had no effect on control littermates (supplemental Fig. 2, available at www.jneurosci.org as supplemental material).

\section{Light-aversive behavior is not attributable to anxiety or differences in motor activity}

To test whether the nestin/hRAMP1 behavior observed in the light-aversion test was influenced by differences in anxiety, we analyzed two fear- or anxiety-related behaviors: predator odorevoked freezing and center avoidance in the open field. At baseline, in the absence of either vehicle or CGRP injection, nestin/ $h R A M P 1$ mice displayed less freezing than control littermates over the entire monitoring period (Fig. 4A), suggesting that hRAMP1 expression might reduce fear. Total time freezing in 10 min was lower in nestin/hRAMP1 mice $(174.55 \pm 21 \mathrm{~s})$ than control mice $(244.25 \pm 14.5 \mathrm{~s} ; p=0.01)$. Overall, there was no difference between predator odor-evoked freezing behavior in nestin/hRAMP1 and control littermates after intracerebroventricular CGRP or vehicle (Fig. $4 B$ ). However, during the first 4 min of the monitoring period, there was a significant increase in

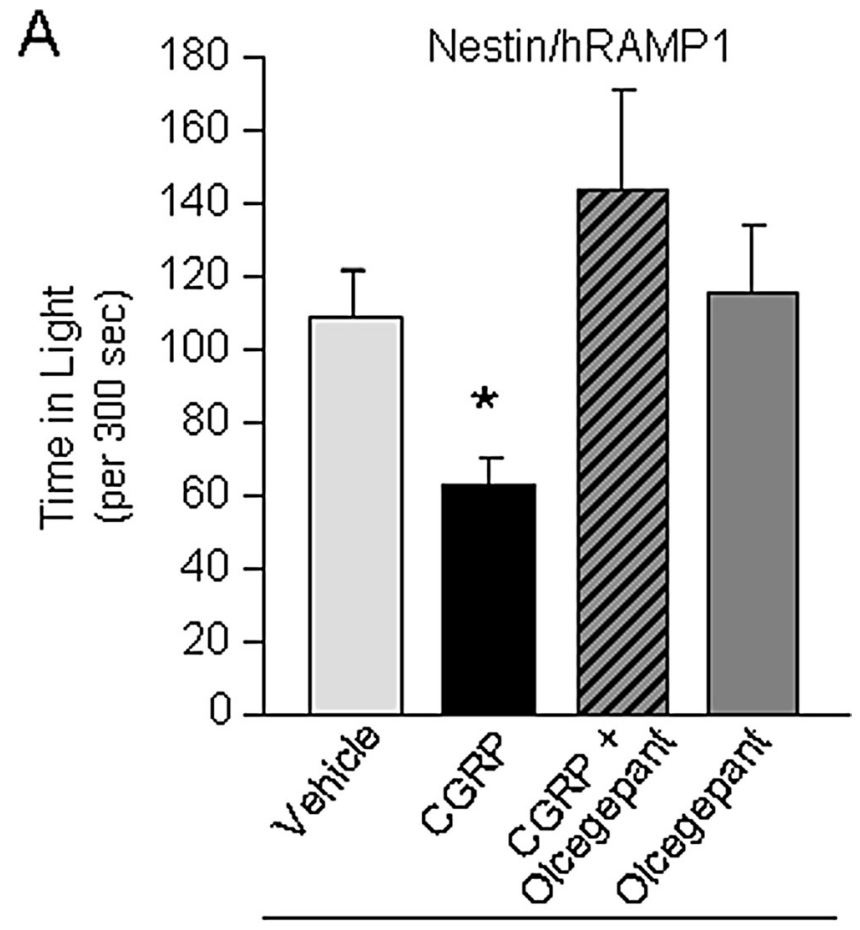

$1^{\text {st }} 300-$ sec interval

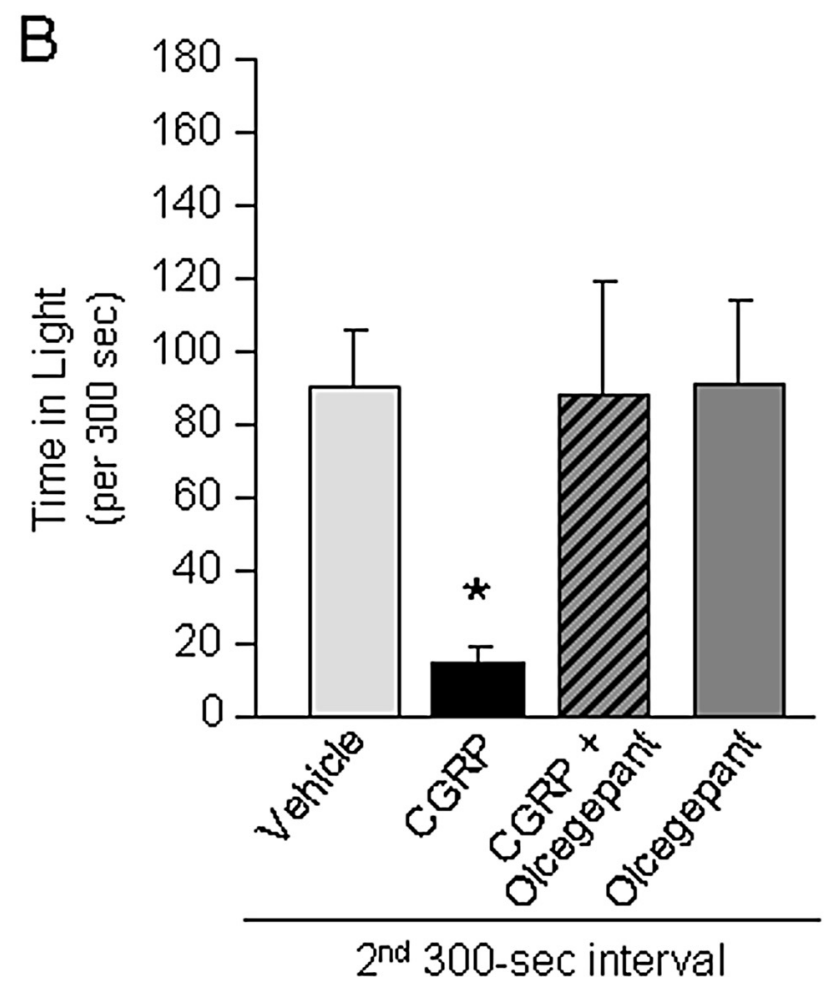

Figure 3. Effect of CGRP receptor antagonist in CGRP-induced light-aversive behavior in nestin/hRAMP1 transgenic mice. $\boldsymbol{A}$, Total time spent in light in the first 300 s interval by nestin/ hRAMP1 mice after intracerebroventricular vehicle (light gray column; $n=12$ ) compared with CGRP (black column; $n=21 ;{ }^{*} p=0.0022$ ) and compared with CGRP plus olcegepant (hatched column; $\left.n=12 ;{ }^{*} p=0.0129\right)$. $\boldsymbol{B}$, Total time spent in light in the second 300 s interval by nestin/hRAMP1 mice after intracerebroventricular vehicle (light gray column; $n=11$ ) compared with CGRP (black column; $n=21 ;{ }^{*} p<0.0001$ ) and compared with (GRP plus olcegepant (hatched column; $n=12 ;{ }^{*} p=0.0259$ ). Both nestin/hRAMP1 and nestinJ/hRAMP1 mice were pooled for these studies, and the vehicle and CGRP treatment data are taken from Figure 2. Error bars indicate SEM. 
freezing behavior induced by CGRP in both nestin/hRAMP1 and control mice. Thus, CGRP increased freezing behavior initially, but the effect was not genotype specific and therefore cannot explain the behavior differences observed in the lightaversion test.

As a second measure of anxiety, we examined the percentage of time spent in the center of an open field over $30 \mathrm{~min}$. Untreated nestin/hRAMP1 mice and control littermates exhibited similar behavior (Fig. $4 C)$. Likewise, neither intracerebroventricular CGRP nor vehicle had any effect on the percentage of time in the center of the field in either genotype (Fig. 4C). We conclude that neither hRAMP1 expression nor CGRP altered anxiety-related behavior in the open field or predator odorinduced freezing assays.

Spontaneous motor activity in the open-field test was similar in untreated nestin/hRAMP1 and control littermates (Fig. 4D). However, after intracerebroventricular treatment with vehicle or CGRP motor activity was decreased in both nestin/hRAMP1 and control mice (Fig. 4D). This was in contrast to the lightaversion chamber (Fig. 2C). CGRP had a more pronounced effect on motor activity of nestin/hRAMP1 mice than littermates in the open field (Fig. 4D).

\section{Discussion}

We have generated a conditional transgenic mouse that is sensitized to CGRP as a potential model to study some of the behavioral aspects of migraine. The rationale for this model was based on the apparent sensitivity of migraineurs to CGRP (Lassen et al., 2002) and the reported efficacy of CGRP antagonists for treating migraine (Olesen et al., 2004; Ho et al., 2008). A key feature of these mice is selective expression of the human RAMP1 subunit in the central and peripheral nervous systems. We have previously reported that RAMP1 is functionally rate limiting in cultures of aortic smooth muscle and trigeminal ganglia (Zhang et al., 2006, 2007). In addition, nestin/hRAMP1 mice show greater CGRP-induced neurogenic inflammation, as measured by plasma extravasation (Zhang et al., 2007), and CGRP-induced mechanical allodynia probably mediated by central sensitization (Marquez de Prado, 2009). To study migraine, we chose to investigate migraineassociated symptoms, since the subjective experience of headache cannot be assessed in rodents. In this report, we have objectively quantified one of the most common nonheadache symptoms of migraine, photophobia.

Nestin/hRAMP1 transgenic mice were more light aversive than littermates. This aversion was greatly enhanced by intracerebroventricular administration of CGRP. Specificity of CGRP action was confirmed by coinjection of the CGRP receptor antagonist olcegepant. Involvement of CGRP was important to establish, because RAMP1 is known to interact with additional
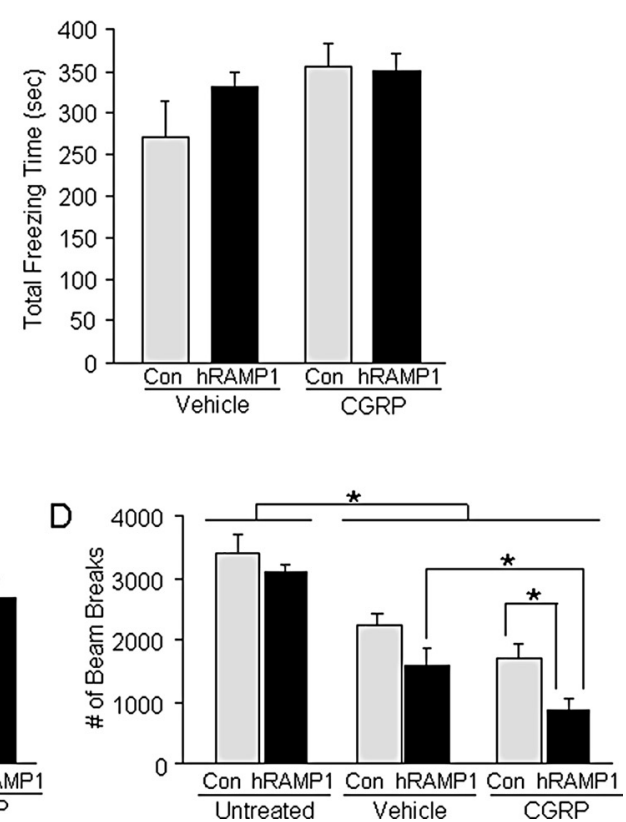

Figure 4. Anxiety and motor activity assessment in nestin/hRAMP1 mice. A, TMT induced freezing behavior in untreated nestin/hRAMP1 mice $(n=8)$ compared with control littermates $(n=9)$. $\boldsymbol{B}$, TMT induced freezing behavior in nestin/hRAMP1 mice after intracerebroventricular vehicle $(n=7)$ and CGRP $(n=9)$, compared with control littermates after intracerebrovenin $10 \mathrm{~min}$. Two-way ANOVA revealed no significant effects over $10 \mathrm{~min}$; however, in the first $4 \mathrm{~min}$, there was a significant main effect of $\operatorname{CGRP}\left(F_{(1,27)}=6.36, p=0.02\right)$, no main effect of genotype $(F<1)$, and no $C$ GRP $\times$ genotype interaction $(F<1)$. $C$ ( $\left.F_{(5,58)}=1.61, p=0.339\right)$. D, Motility in open field by nestin/hRAMP1 and control littermates untreated and after intracerebro0.002 ) and treatment (no injection vs vehicle vs $\left(G R P ; F_{(2,58)}=39.3, p<0.001\right)$ and no genotype by treatment interaction $(F<1)$. Post hoc pairwise comparisons (least significant difference) revealed no difference in motor activity in untreated nestin/hRAMP1 versus control littermates. Significant post hoc comparisons $\left({ }^{*} p<0.05\right)$ are indicated. Error bars indicate SEM.

G-protein-coupled receptors (Hay et al., 2006a). For example, hRAMP1 can interact with the calcitonin receptor to generate the $\mathrm{AMY}_{1(\mathrm{a})}$ amylin receptor, which has a relatively high affinity for CGRP (Hay et al., 2006a). Nevertheless, olcegepant has $~ 150$ fold lower affinity for $\mathrm{AMY}_{1(\mathrm{a})}$ than the CGRP receptor (Hay et al., 2006b). However, given that the drug concentrations at the relevant sites are not known, it is possible that the nestin/hRAMP1 phenotype may reflect a combination of CGRP and AMY 1(a) $_{\text {re- }}$ ceptor actions in the nervous system. Although our results clearly establish an essential role for CGRP in the light-aversive behavior, future studies are needed to identify the mechanisms of CGRP action.

We have demonstrated a novel role for CGRP, but the exact site of action of CGRP remains unknown. CGRP receptors are widely distributed in the nervous system (van Rossum et al., 1997). However, a likely site of action is in the trigeminal nucleus caudalis. Recently, Okamoto et al. (2009) demonstrated that bright light evokes Fos-like immunoreactivity in multiple regions 
of the caudal trigeminal brainstem complex of anesthetized rats, including laminae I-II at the trigeminal caudalis/cervical cord junction region. Hence, CGRP actions in the trigeminal system, as well as perhaps elsewhere in the nervous system, are likely involved in the mechanisms underlying photophobia.

We speculate that the observed light aversion seen in untreated nestin/hRAMP1 mice may be equivalent to the relatively mild photophobia that occurs in migraine patients outside of acute migraine attacks (Main et al., 1997, 2000; Mulleners et al., 2001; Claustrat et al., 2004). A potential mechanism underlying the baseline enhanced light aversion could be the elevated levels of endogenous CGRP we measured in the CSF of nestin/hRAMP1 mice. An elevation of CGRP levels in the nestin/hRAMP1 mice is consistent with our previous data from neuronal cultures demonstrating that CALCA gene transcription is upregulated by CGRP through a self-sustaining positive feedback loop mediated by the CGRP receptor (Zhang et al., 2007). Thus, expression of the $h R A M P 1$ transgene appears to cause a chronic elevation of CGRP synthesis in the nervous system, possibly by an increase in CALCA promoter activity. Interestingly, some studies have reported that plasma CGRP levels are elevated in migraineurs even between attacks (Ashina et al., 2000) and elevation of CGRP in the CSF has been reported in patients with chronic migraine (Sarchielli et al., 2001, 2007; Gallai et al., 2003).

Before concluding that the light aversion reflects photophobia, we first ruled out other causes leading to preference for the dark. We demonstrated that the aversive behavior seen in nestin/ $h R A M P 1$ mice cannot be fully explained by gross abnormality of ocular anatomy, increased general anxiety, or decreased motor activity. Nestin/hRAMP1 mice do not show increased anxietyrelated center avoidance behavior in the open field when compared with control littermates, either untreated or after administration of CGRP. The baseline behavior (uninjected) in the predator odor assay suggests that nestin/hRAMP1 mice were actually less anxious than littermates. Interestingly, CGRP seemed to increase early freezing behavior equally in nestin/hRAMP1 and control mice. Although this suggests that exogenous administration of CGRP may have a subtle effect in the fear response, the effect was comparable in both nestin/hRAMP1 and control mice. These findings corroborate that the light-aversive behavior observed in nestin/hRAMP1 mice is not driven by fear or anxiety.

Likewise, the preference for darkness displayed by nestin/ hRAMP1 mice seems to be attributable to light aversion and not an effect of reduced motility, as shown by the identical spontaneous motor activity during the light-aversion assay. Differences in spontaneous motor activity in the open field and light-aversion tests after intracerebroventricular CGRP administration may be attributable to the possibility of escaping to the dark region only in the latter test. Because animals in discomfort are less spontaneously active, we speculate that the reduced activity observed in the intracerebroventricularly injected groups in the open field may be attributable to cranial discomfort and/or absence of escape. The greater reduction in activity in nestin/hRAMP1 mice treated with CGRP may reflect more severe discomfort. In fact, one of the diagnostic criteria of migraine is aggravation of the headache by routine physical activity (walking or climbing stairs) (Headache Classification Subcommittee of the International Headache Society, 2004). Hence, we believe that the lightaversive behavior represents an objective and measurable parameter equivalent to photophobia. Although caution is required when comparing rodent and human behavior, we believe that the stimulus leading the mice to avoid the light must be quite unpleasant to overcome their innate and strong exploratory in- stinct. This is the first study to indicate that migraine symptoms are not unique to humans and may be experimentally induced in rodent models.

Our findings suggest that a single gene, RAMP1, is a modifier of photophobia and by extension migraine. A genetic or epigenetic elevation of RAMP1 levels provides a mechanism for the reported sensitivity to CGRP of migraineurs (Lassen et al., 2002; Petersen et al., 2005). RAMP1 is known to confer species specificity to the CGRP receptor; thus, rodents have previously been an inadequate model for the development of CGRP receptor antagonists for humans. By expressing human RAMP1, nestin/ hRAMP1 mice offer a useful tool for future drug development. We conclude that hypersensitivity to CGRP attributable to elevated hRAMP1 could contribute to migraine susceptibility. Furthermore, nestin/hRAMP1 mice may represent a model for the development of novel therapeutic strategies and mechanistic studies of migraine and other conditions associated with photophobia.

\section{References}

Amini A, Digre K, Couldwell WT (2006) Photophobia in a blind patient: an alternate visual pathway. Case report. J Neurosurg 105:765-768.

Arulmani U, Maassenvandenbrink A, Villalón CM, Saxena PR (2004) Calcitonin gene-related peptide and its role in migraine pathophysiology. Eur J Pharmacol 500:315-330.

Ashina M, Bendtsen L, Jensen R, Schifter S, Olesen J (2000) Evidence for increased plasma levels of calcitonin gene-related peptide in migraine outside of attacks. Pain 86:133-138.

Bahra A, May A, Goadsby PJ (2002) Cluster headache: a prospective clinical study with diagnostic implications. Neurology 58:354-361.

Benemei S, Nicoletti P, Capone JA, Geppetti P (2007) Pain pharmacology in migraine: focus on CGRP and CGRP receptors. Neurol Sci 28 [Suppl 2]:S89-S93.

Claustrat B, Brun J, Chiquet C, Chazot G, Borson-Chazot F (2004) Melatonin secretion is supersensitive to light in migraine. Cephalalgia 24:128-133.

Crawley J, Goodwin FK (1980) Preliminary report of a simple animal behavior model for the anxiolytic effects of benzodiazepines. Pharmacol Biochem Behav 13:167-170.

Doods H, Hallermayer G, Wu D, Entzeroth M, Rudolf K, Engel W, Eberlein W (2000) Pharmacological profile of BIBN4096BS, the first selective small molecule CGRP antagonist. Br J Pharmacol 129:420-423.

Drummond PD, Woodhouse A (1993) Painful stimulation of the forehead increases photophobia in migraine sufferers. Cephalalgia 13:321-324.

Gallai V, Alberti A, Gallai B, Coppola F, Floridi A, Sarchielli P (2003) Glutamate and nitric oxide pathway in chronic daily headache: evidence from cerebrospinal fluid. Cephalalgia 23:166-174.

Goadsby PJ, Edvinsson L (1993) The trigeminovascular system and migraine: studies characterizing cerebrovascular and neuropeptide changes seen in humans and cats. Ann Neurol 33:48-56.

Goadsby PJ, Edvinsson L (1994) Human in vivo evidence for trigeminovascular activation in cluster headache. Neuropeptide changes and effects of acute attacks therapies. Brain 117:427-434.

Goadsby PJ, Edvinsson L, Ekman R (1990) Vasoactive peptide release in the extracerebral circulation of humans during migraine headache. Ann Neurol 28:183-187.

Goadsby PJ, Lipton RB, Ferrari MD (2002) Migraine-current understanding and treatment. N Engl J Med 346:257-270.

Hallett M, Evinger C, Jankovic J, Stacy M (2008) Update on blepharospasm: report from the BEBRF International Workshop. Neurology 71:1275-1282.

Hay DL, Poyner DR, Sexton PM (2006a) GPCR modulation by RAMPs. Pharmacol Ther 109:173-197.

Hay DL, Christopoulos G, Christopoulos A, Sexton PM (2006b) Determinants of 1-piperidinecarboxamide, N-[2-[[5-amino-1-[[4-(4-pyridinyl)1 - piperazinyl $]$ carbonyl $]$ pentyl $]$ amino $]-1$ - [(3,5 - dibromo - 4 - hydroxyphenyl)methyl] -2-oxoethyl]-4-(1,4-dihydro-2-oxo-3(2 H)-quinazolinyl) (BIBN4096BS) affinity for calcitonin gene-related peptide and amylin receptors-the role of receptor activity modifying protein 1. Mol Pharmacol 70:1984-1991. 
Headache Classification Subcommittee of the International Headache Society (2004) The international classification of headache disorders: 2nd edition. Cephalalgia 24 [Suppl 1]:9-160.

Ho TW, Ferrari MD, Dodick DW, Galet V, Kost J, Fan X, Leibensperger H, Froman S, Assaid C, Lines C, Koppen H, Winner PK (2008) Efficacy and tolerability of MK-0974 (telcagepant), a new oral antagonist of calcitonin gene-related peptide receptor, compared with zolmitriptan for acute migraine: a randomised, placebo-controlled, parallel-treatment trial. Lancet 372:2115-2123.

Irimia P, Cittadini E, Paemeleire K, Cohen AS, Goadsby PJ (2008) Unilateral photophobia or phonophobia in migraine compared with trigeminal autonomic cephalalgias. Cephalalgia 28:626-630.

Juhasz G, Zsombok T, Jakab B, Nemeth J, Szolcsanyi J, Bagdy G (2005) Sumatriptan causes parallel decrease in plasma calcitonin gene-related peptide (CGRP) concentration and migraine headache during nitroglycerin induced migraine attack. Cephalalgia 25:179-183.

Kowacs PA, Piovesan EJ, Werneck LC, Tatsui CE, Lange MC, Ribas LC, da Silva HP (2001) Influence of intense light stimulation on trigeminal and cervical pain perception thresholds. Cephalalgia 21:184-188.

Lassen LH, Haderslev PA, Jacobsen VB, Iversen HK, Sperling B, Olesen J (2002) CGRP may play a causative role in migraine. Cephalalgia 22:54-61.

Lipton RB, Bigal ME, Diamond M, Freitag F, Reed ML, Stewart WF (2007) Migraine prevalence, disease burden, and the need for preventive therapy. Neurology 68:343-349.

Main A, Dowson A, Gross M (1997) Photophobia and phonophobia in migraineurs between attacks. Headache 37:492-495.

Main A, Vlachonikolis I, Dowson A (2000) The wavelength of light causing photophobia in migraine and tension-type headache between attacks. Headache 40:194-199.

Mallee JJ, Salvatore CA, LeBourdelles B, Oliver KR, Longmore J, Koblan KS, Kane SA (2002) Receptor activity-modifying protein 1 determines the species selectivity of non-peptide CGRP receptor antagonists. J Biol Chem 277:14294-14298.

Marquez de Prado B, Hammond DL, Russo AF (2009) Genetic enhancement of calcitonin gene-related peptide-induced central sensitization to mechanical stimuli in mice. J Pain, in press.

McLatchie LM, Fraser NJ, Main MJ, Wise A, Brown J, Thompson N, Solari R, Lee MG, Foord SM (1998) RAMPs regulate the transport and ligand specificity of the calcitonin-receptor-like receptor. Nature 393:333-339.
Mulleners WM, Aurora SK, Chronicle EP, Stewart R, Gopal S, Koehler P (2001) Self-reported photophobic symptoms in migraineurs and controls are reliable and predict diagnostic category accurately. Headache 41:31-39.

Okamoto K, Thompson R, Tashiro A, Chang Z, Bereiter DA (2009) Bright light produces fos-positive neurons in caudal trigeminal brainstem. Neuroscience 160:858-864.

Olesen J, Diener HC, Husstedt IW, Goadsby PJ, Hall D, Meier U, Pollentier S, Lesko LM (2004) Calcitonin gene-related peptide receptor antagonist BIBN 4096 BS for the acute treatment of migraine. N Engl J Med 350:1104-1110.

Petersen KA, Lassen LH, Birk S, Lesko L, Olesen J (2005) BIBN4096BS antagonizes human alpha-calcitonin gene related peptide-induced headache and extracerebral artery dilatation. Clin Pharmacol Ther 77:202-213

Sarchielli P, Alberti A, Floridi A, Gallai V (2001) Levels of nerve growth factor in cerebrospinal fluid of chronic daily headache patients. Neurology 57:132-134.

Sarchielli P, Pini LA, Coppola F, Rossi C, Baldi A, Mancini ML, Calabresi P (2007) Endocannabinoids in chronic migraine: CSF findings suggest a system failure. Neuropsychopharmacology 32:1384-1390.

Tfelt-Hansen P, Le H (2009) Calcitonin gene-related peptide in blood: is it increased in the external jugular vein during migraine and cluster headache? A review. J Headache Pain 10:137-143.

Tronche F, Kellendonk C, Kretz O, Gass P, Anlag K, Orban PC, Bock R, Klein R, Schütz G (1999) Disruption of the glucocorticoid receptor gene in the nervous system results in reduced anxiety. Nat Genet 23:99-103.

Tvedskov JF, Lipka K, Ashina M, Iversen HK, Schifter S, Olesen J (2005) No increase of calcitonin gene-related peptide in jugular blood during migraine. Ann Neurol 58:561-568.

van Rossum D, Hanisch UK, Quirion R (1997) Neuroanatomical localization, pharmacological characterization and functions of CGRP, related peptides and their receptors. Neurosci Biobehav Rev 21:649-678.

Zhang Z, Dickerson IM, Russo AF (2006) Calcitonin gene-related peptide receptor activation by receptor activity-modifying protein-1 gene transfer to vascular smooth muscle cells. Endocrinology 147:1932-1940.

Zhang Z, Winborn CS, Marquez de Prado B, Russo AF (2007) Sensitization of calcitonin gene-related peptide receptors by receptor activitymodifying protein-1 in the trigeminal ganglion. J Neurosci 27:2693-2703. 